\title{
Das Böse - ein Machwerk der Bestie
}

K. Mohtadi

Korrespondenz:

Dr. med. K. Mohtadi Kantonale Psychiatrische Klinik CH-4915 St. Urban
«Eins, zwei, drei, um Mitternacht, sechs, sieben, acht, sie werden wach, neun, zehn, elf, zwölf, sie geistern umher. Sie kommen und gehen um Mitternacht. Die Leichen haben ins Bett gemacht. Da wackelt das Gebiss und die Leichen tanzen im Mondschein Rock'n'roll.» Dann hörte sie auf zu singen und fragte unvermittelt: «Papa, warum hilft die Lehrerin jenen Schülerinnen, die sowieso in der Klasse keine Mühe haben, und für die, welche diese nötig haben, keine Geduld aufbringt?» Während Ibis mit ihrer lispelnden Zunge bei jedem Mitgefühl erwecken sollte, werde ihr Beistand vorenthalten und beim Nachfragen übergangen, obschon sie weiss und blond sei, werde sie sonst auch wegen ihres Hörgeräts gehänselt. Auch Maria werde beleidigt. Mitten ins Gesicht sagen sie ihr: «Du stinkst!» «Diese sind nun meine Freundinnen. Ich kann und will aber nicht mehr zu ihnen halten, da man sonst mich mit ihnen in ihren Spott einbezieht oder einfach ignoriert. Ich muss unter der Schwäche meiner Freundinnen nur leiden. Ich werde mich zu denen gesellen, die stark sind, und um die sich alle scharen und werde meine Ruhe haben! Bei der Schulfeier verkündete man vor hundert Schülerinnen und Schülern auf dem Schulplatz, dass der U. das nächste Jahr nach Reiden gehen werde, und jeder wusste, was damit gemeint war. Manche lachten, dem mageren, grossgewachsenen U. sammelten sich Tränen in den Augen. $\mathrm{Ob}$ das notwendig gewesen sei, sein schulisches Ungenügen publik zu machen und ihn vor den Mitschülerinnen so blosszustellen?»

Der Papa fühlte sich betreten, irgendwie verblüfft und hilflos. Er hielt kurz den Atem an, schüttelte in «der Seele» den Kopf und starrte auf den Boden, um der Tochter nicht in die Augen zu schauen. Wie traurig das alles ist, dachte er, schon in diesem Alter aufzugeben und unter Druck die Seite zu wechseln. Identifikation und Verbundenheit mit dem Aggressor ist ja eine mögliche Abwehr der Erwachsenen; sie sollte eher verdrängen. Sie schien aber eine pragmatische, wenn auch nicht ehrenvollere Lösung für ihre Probleme gefunden zu haben.

Sie wusste nicht, was er gerade las: von Helden, die mit Wunden bedeckte Frauen erwürgten, die noch im Sterben ihre Kinder an die blutenden Brüste pressten. «Mädchen mit aufgeschlitzten Bäuchen hauchten ihre letzten Seufzer aus, nach dem einige Helden ihre natürlichen
Bedürfnisse an ihnen befriedigt hatten. Andere, die halb verbrannt waren, flehten schreiend um den Gnadenstoss.» (aus Voltaires «Candide oder Die beste der Welt»).

Es sei halt nicht so ungewöhnlich, dass die Jugendlichen Gruppen bildeten und unter anderem auch über Schwächere herfallen, das sei vorübergehend, gab er zur Antwort, konnte sich aber einen weiteren Kommentar nicht ersparen: deine Freundinnen haben keine Eltern mit Ansehen und Einfluss in der Gemeinde. Wenn Ibis auch keine Tochter eines Asylanten sei und ihre blonden Haare wie eine Mähne aus Seide glänzen, sei sie doch eine Ausländerin, behindert durch die Zunge, das Gehör und mit einem Vater als Gastarbeiter aus einem kriegszerrütteten Land. Und als solche fühle man sich ihr gewachsen und manche sich berechtigt, ihre vermeintliche Vorzugsstellung ihr gegenüber auszuleben. Maria sei zwar keine Ausländerin, sie sei aber schon körperlich schmächtig und als Tochter eines armen Pächters und einer kranken Mutter ohne Rückhalt, so dass sie leicht Aggressionsobjekt der Frustrierten - das bedeute so ungefähr «zu kurz gekommen» - werden könne. Sie hassen sich selbst und überwinden ihre eigenen Schwächen dadurch, dass sie euch anpöbeln. Dabei fühlen sie sich wohl; eine findet Geltung vor den Anwesenden, der andere Akzeptanz. Man bezeichne Maria als Umweltverschmutzung und tituliere sie mit «Drecksau», da man annehme und im Glauben sei, sie wäre nicht imstande, sich zu wehren, und aus diesem Grund sie und ihre Eltern auch verachten. Dies alles sei aber vorübergehend, nächstes Jahr würden alle ein Jahr älter sein, und vernünftiger. Vielleicht gebe es eine neue Zusammensetzung der Klasse und ihr bekommt eine verständnisvollere, ältere, lebenserfahrene Lehrerin, eine Mutter! Man werde schon einmal auf die Idee kommen, die frisch diplomierten Lehrerinnen eher in den mittleren Klassen und nicht in den unteren einzusetzen. Die, welche euch heute schikanieren, werden morgen mit Problemen und Sorgen konfrontiert, unter denen sie das Genick brechen und vielleicht selber Spottobjekte anderer werden. Sie malt, er schweigt!

Wie gerne, ja begierig er Wörter sammelte, die das Leiden und die Schmerzen beschreiben: Trübsinn, Mühsal, Gram, Erniedrigung, Ablehnung, Ignoranz, peinigen, drangsalieren, ver- 
achten, Habgier ... morden. Und nun fingen die Wörter in seinem Gehirn an, zu oszillieren. Dabei riss sich eine Strophe aus dem Gedächtnis los und wurde laut: «Hätte das Leiden einen Rauch wie das Feuer, finster wäre die Welt für Ewigkeit, suchtest Du auf Erden, fändest Du keinen einzigen fröhlichen Gelehrten.»

Obschon die Geschichte des Schmerzes sehr alt ist, assoziierte er damit den Charles Darwin. Er hätte die Leidensgeschichte der Freundinnen von Mina wissenschaftlich begründet und legitimiert: Nur der Kräftige, der Tüchtige hat eine Daseinsberechtigung, alle Lebens- und Arbeitsuntüchtigen sind zum Verschwinden verurteilt und als solche wohl verachtungswürdig. Und diejenigen, die diese in Schutz nehmen, stemmen sich gegen die Natur und Gesetze der Schöpfung. Man ist demnach auch berechtigt, sie genau so wie ihre Schutzbefohlenen zu behandeln. Der Schwache ist von Natur aus gekennzeichnet und gebrandmarkt. Der Darwin hat dieses biologische Gesetz bei Tieren beschrieben und demonstriert. Und dies ist für die Menschen folgerichtig und ein Imperativ für dessen orthodoxe Anhängerschaft, gemäss dieser Regel zu handeln. Bemühungen der grossen Geister der Menschheitsgeschichte, aus dem Säugetier Mensch einen Menschen zu machen, hin oder her!

Der liebe Gott schuf die Menschen aus einem Stoff, rüstete sie aber verschieden aus, das Leben zu bewältigen oder eben auch nicht. Er hat den Menschen unverbindlich und ohne Zusicherung Zähne gegeben, dass diese auch einmal zum Essen eines Stück Brotes oder vielleicht Fleisches gebraucht werden können. Er schickt Dürre und Krankheit, lässt die Menschen im Elend verhungern, dann sitzt er unbekümmert zur Schau jenes ausgemergelten, afrikanischen Buben, der sich mit eingesunkenen Wangen, lichtlosen, hohlen Augen und einem Wasserbauch - mit ganzem vorausbestimmten Leid im Gesicht bemüht, vom Stamm eines lange verdorrten Baumes ohne Krone einen Brocken Zellulose abzubeissen, den er zu kauen gar nicht mehr mächtig wäre, und vor unseren Augen im Fernsehen erlischt. Auf dem Schirm nebenan betrachtet der gleiche Schöpfer die Street Parade mit hüpfenden Beinen, wiegenden Hüften und - soweit das Auge reicht - begehrlichem Fleisch und warmem Schweiss.

Als er sagte: «Gott ziere manche Menschen mit Haaren, etliche aber mit Wolle», unterbrach ein entsetzter Aufschrei den Rapport. Gestreckte Hände der ohnmächtigen, weinenden Mütter, deren Kinder an ausgetrockneten, atrophierten Brüsten hängen oder ermordet am Rande der
Strassen liegen, bewirken bei ihrem Schöpfer nicht das geringste. Die fromme, liebenswürdige Kollegin, welche auf einer indonesischen Insel auf einer Missionsstation gearbeitet hatte, protestierte - ihn zugleich warnend -, als seine Gedanken laut ausgesprochen wurden: «Besässe Gott bei soviel Leid auf der Erde ein Gewissen, hätte er sich schon dutzendmal erhängt für das begangene Verbrechen, Menschen erschaffen zu haben. 〈Haben Sie Angst!〉 sagte sie, ১Jesus Christus liebt die Menschen», worauf er sie mit der Bemerkung beruhigte, dass er jenen Gott meine, der ihm einmal mit Liebe und Milch vorgestellt worden sei, und der sei kein Jesus Christus. Hätte er selber einen Vater namens Jusef gehabt - seine Mutter hiess Mariam - hiesse er heute Issa, also Jesus!»

Diese Gedanken befassen sich auch nicht mit der Religion, sondern setzen sich philosophisch mit dem Rätsel Mensch auseinander. Jeder, der die Voraussetzung mitbringe, irgendwann ein Gewissen zu entwickeln, werde religiös geboren, und beschäftige man sich mit der Natur, müsse man den Schöpfer bewundern. Die Kollegin gab sich zufrieden.

«In der Geschichte Panamas gebe es weder Helden noch Epen, ihre Erniedrigung zelebrieren sie als Befreiung», schrieb ein Journalist. Woher die Menschen soviel Kraft aufbringen, welche Hartnäckigkeit und Zivilcourage hat die Biologie, welche Zumutung ist das Leben für dessen Träger, fragt man sich. «Das Brot hat von Anfang an eine minimale Bedeutung, das Wort Gottes ist von Bedeutung. Die irdische Gerechtigkeit besteht in Gleichheit und Berechtigung der Menschen den Gott zu verehren.» Theologie der Befreiung zielt auf Verwirklichung der sozialen Gerechtigkeit auf Erden und impliziert damit die «marxistische Ideologie» und Gottesverneinung. Eine Trost bleibt immerhin für die Völker, die ihr Weinen in «Trauerliedern» singen können!

Konfrontiert und vergesellschaftet mit Krankheit, Sterben und Tod denkt man oft an Jenseits oder unter dem Erdseits. Wie hat sich Darwin seine Zukunft, die jenseitige mit der Beschreibung von Artenselektion und deren eugenischen und sozialen Folgen gestaltet, angenommen, es gebe ein Jenseits, wo er denn wohl gelandet sei? Die gleiche Frage wäre im Falle von Thomas Edison leicht zu beantworten. Für Nobel schrieb ein offensichtlich wütender Schriftsteller: «Der Weg nach Stockholm führe über Tel Aviv und «der Nobel〉 verbrenne im Feuer der Hölle bis in die Ewigkeit.»

Während das Paradies unter Verwaltung Gottes steht, wird die Hölle durch Satan geleitet. 
Nun, die Entdeckung, die er glaubte, gemacht zu haben, war, dass diese beiden Herrschaften diametral zur Vorstellung Zarathustras eng miteinander kooperieren und gemeinsam am Gedeih und Verderb der Welt beteiligt sind. In der Tat war er der Meinung, dass der eine ohne den anderen gar keine Bedeutung, ja keine Funktion haben könnte. Und nachdem er sich zwei Persönlichkeiten vom Schlage des Schöpfers und des Vernichters nicht vorstellen vermag, gelangte er zur Auffassung, dass diese ein und dieselbe Person darstellen. Will man in der Natur eine Rationalität und absolute Vernunft erkennen, muss man diese Attribute den beiden Akteuren oder eben einer Verantwortlichen zubilligen. In dem Sinne sind Erdbeben, Überschwemmungen und andere Naturkatastrophen zielgerichtete, den Lebewesen übergeordneten Homöostase dienende, quasi die gestörte Gerechtigkeit wieder herstellende, von absoluter Vernunft gesteuerte Aktionen.

Dieser Akteur hat die Menschen nach seinem Abbild geschaffen. Überträgt man diese Überlegungen auf die menschlichen Belange, wird der Selbsterhaltungstrieb dem Göttlichen zugerechnet, Leid und Krankheiten dem Teuflischen. Die Geburt und das Sterben werden in ihren verschiedenen Erscheinungsformen und Metamorphosen wechselnde Prioritäten der Geltung dieser Kräfte in einer in sich geschlossenen Bahn sein. Der Kampf um die Erhaltung des eigenen Reviers, die Vertreibung des Feindes oder dessen Vernichtung sind in diesem Fall eine gemeinsame Funktion der konstruktiven und destruktiven Kräfte, die - gegen Perversion allerdings nicht gefeit - eng miteinander einem Ziel, der Sicherheit eines Menschen oder einem Klüngel dienen, während der Feind in diesem Geschehen den destruktiven Kräften anheimfällt.

Der Umstand entzieht sich dem Walten und der Kontrolle der entscheidenden Instanz und wird ihr fremd, wenn das Handeln der Menschen den rationalen Sinn verliert: ein russischer Offizier lässt nach Überwinden seines vermeintlichen Gewissens - der Träger moralischer Werte und göttlicher Verbote - um Mitternacht eine minderjährige Töchter einer tschetschenischen Familie holen und erwürgt sie, nachdem er sie vergewaltigt hat. Die Polizisten schlagen einen bewusstlosen Schwarzen zu Tode. Andere erschlagen einen obdachlosen Lumpen. Die Dynamik dieser Phänomene findet man in harmloserer Gestalt in der Verachtung, Erniedrigung und Demütigung der Mitmenschen. Denn diese formen sich aus der gleichen Matrix und beziehen ihre Energie aus derselben Quelle. Ferner werden sie durch ausschliesslich rohe Affekte verschiedener Intensität begleitet. Diese Übersteigerungen, diese der Rationalität und Ökonomie des Daseins abweichenden Verhaltensweisen sind gemessen an unabdingbarer Sinnhaftigkeit der Natur, zu der auch der Mensch gezählt wird, demzufolge weder göttlicher noch satanischer Herkunft, denn diese machen die absolute Vernunft aus. Wenn die menschliche Entwicklung Freudscher Vorstellung darin besteht, die Triebe in Ich umzuwandeln, woraus sich dann durch Erziehung ein Über-Ich ausbilden soll, muss man die Ausschläge jener Dynamik in einer überwiegend intelligenzquotientunabhängigen, ontogenetisch bedingten, von urtümlichen Trieben gesteuerten Formation des Ichs mit rudimentären, mangelhaft differenzierten Affekten bei einem verkümmerten Über-Ich suchen. Diese psychologische Gestalt nannte er «die Bestie», deren Werk «das Böse» ist. Zur Eigenheit dieser Bestie gehört eine Selbstwertschätzung, die aufrechterhalten wird durch die Gewissheit, über ein überlegenes Tötungs- beziehungsweise Gewaltpotential zu verfügen, das jederzeit und beliebig oft, mitunter mit moralischem Anhauch gegen einen Unterlegenen, der je nach Notwendigkeit auch dämonisiert wird, eingesetzt werden kann.

Bei diesen Überlegungen und menschlichen Anmassungen, Herausforderungen und Übermass an Anspruch den Artgenossen und der Welt gegenüber stellte sich für ihn die Frage, welche Funktion eigentlich diesem Lebewesen, dem Menschen, als Teil der Natur zukommt und was er als Gegenleistung für die Dienste des Wassers und des Windes, der Erde und der Sonne anzubieten hat? Was rechtfertigt seine Existenz auf der Erde?

Ein weiterer Protest meldete sich bei der Formulierung, dass die Funktion des Menschen darin liege, ein Wirt der Bakterien, Viren, Pilze und eventuell Würmer zu sein und Düngemittel zu produzieren. Der Vorsatz, durch Aneignung göttlicher Adjektive, sich zu einem Menschen göttlicher Prägung zu entwickeln und diese mittels genetischer Information weiterzugeben, bis der Mensch die Stufe der Vollendung erreicht, kann ein teleologisch-entelechisches Ziel sein, um aus dem Leben einen Sinn abzugewinnen, ein Beitrag des Menschen zum Funktionieren der Welt ist es nicht!

Das weitere Kopfzerbrechen kann überflüssig erscheinen, wenn man sich bewusst wird, dass dem Menschen jene Bedeutung nicht zukommt, die er sich zuschreibt, nämlich vom Schöpfer geliebt oder bestraft zu werden, gerade als ungeheuerlich muss die Vorstellung erscheinen, der Gott erwarte und benötige vom Menschen 
1 Mohammad è Gasali ( ${ }^{\star} 1058$ bei Tus in Khorasan, Iran). Elixier der Glückseligkeit. Herausgegeben von Walter Otto. Jena: Eugen Dietrichs; 1923.
Ergebenheit, Achtung und Verehrung. Der Mensch scheint ja auch für die Existenz und den Weiterbestand der Welt nicht die geringste Bedeutung zu haben, eher interessant als absurd dünkt in diesem Zusammenhang der Slogan jener, die die spätere Geburt des Propheten Mohammeds als Anlass der Schöpfung verkaufen wollen.

Die entscheidenden Regeln und Gesetze der Natur berühren den Menschen doch höchstens dann, wenn er bei der Wiederherstellung der Homöostase und Ordnungsverhältnisse in der Natur, wie beispielsweise bei einem Erdbeben oder einer Überschwemmung, umkommt, was man als Betriebsunfall betrachten konnte, denn, wo gehobelt wird, fallen Späne.

Damit kann man allerdings den sich aufdrängenden Widerspruch nicht aus dem Weg gehen, und zwar der hochentwickelten, real existierenden Anatomie und Physiologie des Menschen, die in keiner Weise für die ihm hier unterstellten Funktionen erforderlich wären. Verlockend scheint - nachdem der Mensch über die Geburt und den Tod der Tiere entscheidet und womöglich die Vegetation pflegt - die Hypothese, dem Menschen würden als Vertreter Gottes Aufgaben auf der Erden anvertraut.

Mit dieser Hypothese konnte sich die Kollegin anfreunden, neugierig wurde sie dennoch auf jenen Gott, von dem er sprach: «Lob und Preis gleich der Zahl der Sterne des Himmels, der Tropfen des Regens, der Blätter der Bäume, der Sandkörner der Wüste, der Atome der Erde und des Himmels sei dem einigen Gott, der da ist herrlich und gewaltig, gross und erhaben, ruhmvoll und voll Glanzes, dessen vollkommene Herrlichkeit kein Geschöpf begreifen und dessen wahres Wesen niemand erkennen kann, denn er selbst. Denn das Geständnis der Ohnmacht, ihn in Wahrheit zu erkennen, ist die letzte Erkenntnis der Aufrichtigen, das Bekenntnis des Unvermögens, ihn nach Gebühr zu loben und zu preisen, der höchste Lobpreis der Engel und Propheten. Erschrecken über die ersten Strahlen seiner Herrlichkeit ist die letzte Grenze alles Verstandes der Verständigen, verwirrtes, bestürztes Erschauern das äusserste Ziel, das die 〈Wegschreiter〉 und Jünger〉, die seiner Schönheit Nähe suchen, erreichen [...].» [1].

Im Luzerner Planetarium projizierte man schliesslich den Planet Erde auf den Himmel. Ein kleiner Stern unter unzähligen Sternen und kommentierte, dass der Durchmesser unseres Planeten im Vergleich zum jenen des Universums eine Haarbreite darstelle. Er wies den kleinen Sohn auf den Planet Erde hin und sagte: «Lueg! Döt esch d'Ärde, wo mer deheime send», worauf dieser ihn fragte: «Wo esch de Sankt Orbe?» 